\title{
Room temperature evolution of gold nanodots deposited on silicon
}

\author{
C. Garozzo • A. Filetti • C. Bongiorno • A. La Magna • \\ F. Simone $\cdot$ R. A. Puglisi
}

Published online: 9 May 2014

(C) The Author(s) 2014. This article is published with open access at SpringerLink.com

\begin{abstract}
In this work, the morphological and structural evolution of gold nanodots deposited on Si substrates has been monitored for $2.4 \times 10^{3} \mathrm{~h}$. Gold nanodots on $\mathrm{Si}$ are of great scientific interest because they can be used in numerous ways, for example as subwavelength antennas in plasmonics, as electrical contacts in nanometric devices, or as catalysts for the formation of quasi-1dimensional nanostructures. Their characteristics have been studied in a very large number of papers in literature, and among the several aspects, it is known that continuous $\mathrm{Au}$ films peculiarly interact with $\mathrm{Si}$ by interdiffusion even at room temperature. It would be expected that also small nanostructures could undergo to an interdiffusion and consequent modifications of their structure and shape after aging. Despite the cruciality of this topic, no literature papers have been found showing a detailed morphological and structural characterization of aged Au nanodots. Au nanoparticles have been deposited by sputtering on Si and stored in air at temperature between 20 and $23{ }^{\circ} \mathrm{C}$ and humidity of about $45 \%$, simulating the standard storage conditions of most of the fabrication labs. The morphological and structural characterizations have been performed by bright field transmission electron microscopy (TEM). A specific procedure has been used in order to avoid any modification of the material during the specimen preparation for the TEM analysis. A digital processing of the TEM images has allowed to get a large statistical analysis on the particles size distribution. Two
\end{abstract}

C. Garozzo $\cdot$ A. Filetti $\cdot$ C. Bongiorno $\cdot$ A. La Magna $\cdot$

R. A. Puglisi $(\bowtie)$

Consiglio Nazionale delle Ricerche, Istituto per la Microelettronica e

Microsistemi, Strada Ottava 5 Zona Industriale, 95121 Catania, Italy

e-mail: rosaria.puglisi@imm.cnr.it

F. Simone

Dipartimento di Fisica e Astronomia, Università di Catania, Via

Santa Sofia 64, 95123 Catania, Italy different types of nanoparticles are found after the deposition: pure gold crystalline nanodots on the Si surface and gold amorphous nanoclusters interdiffused into the $\mathrm{Si}$ subsurface regions. While the nanodots preserve both morphology and structure all over the time, the amorphous agglomerates show an evolution during aging in morphology, structure, and chemical phase.

Keywords Interfaces $\cdot$ Gold $\cdot$ Silicon $\cdot$ Nanodots Transmission electron microscopy $\cdot$ Aging

\section{Introduction}

Gold nanodots deposited on silicon are of significant importance because this material presents a large number of applications like as subwavelength antennas in plasmonic applications, or as catalysts for the growth of silicon nanowires (Si-NWs), or even as electrical contacts in Si nanodevices. In the first example, the gold nanoparticles are exploited to increase light absorption in solar cells, thanks to the surface plasmon resonances, when they are in contact with the active surface of semiconductor. But most of the experimental results [1-3] demonstrate that these mechanisms, and consequently the cell performance, are strongly dependent on the geometrical characteristics of the dot, such as size and shape. Another field of application for gold nanodots is in the synthesis of Si-NWs, where they can be used either as catalyst seeds in a bottom-up approach like the vapor-liquid-solid process, or as etching initiator in a topdown process like the metal chemical etching [4-8]. Also, in these cases, the main morphological characteristics of the final Si-NWs such as diameter and growth (/etching) direction are determined by the size of the catalyst and their interconnection, i.e., by the original Au dot characteristics [9-12]. The SiAu system has been also studied from the point of view of the electrical stability in metallic contacts. Akhtari-Zavareh and 
coworkers [13] recently studied the morphological and electrical characteristic of an $\mathrm{Au} / \mathrm{Si}$ diode. They found that after air exposure there is a formation of a continuous interfacial layer due to an interaction between $\mathrm{Au}$ and $\mathrm{Si}$. This surface or interfacial interaction leads to the degradation of the rectifying diode properties.

The evidence of an interaction between gold and silicon is shown in many works for several annealing temperatures. At $T=150{ }^{\circ} \mathrm{C}$, i.e., below the $\mathrm{Au}-\mathrm{Si}$ eutectic point $\left(363{ }^{\circ} \mathrm{C}\right)$, Rutherford backscattering spectrometry (RBS) analysis demonstrated that in a Si sample covered with an evaporated gold layer, Si atoms migrate through the $\mathrm{Au}$ film, accumulate on its surface, and oxidizes after air exposure [14]. Room temperature evolution of continuous Au layers evaporated on $\mathrm{Si}$ (111) has been investigated too [15]. The gold films were evaporated in this case at $T<50^{\circ} \mathrm{C}$ on clean silicon and characterized in situ by using electron loss spectroscopy and Auger electron spectroscopy. The interacted regions have been identified as metastable alloys or silicides [16, 17]. A critical thickness below which the gold seems inert and not able to create alloying has been identified, and, if it is less than $2 \mathrm{ML}$, gold and $\mathrm{Si}$ do not intermix even at temperatures as high as $800^{\circ} \mathrm{C}$ [15]. For a thickness larger than $5 \mathrm{ML}$, Au reacts with silicon also at room temperature [15]. The critical thickness has been in this case estimated as the plateau exhibited by the growth rate measured by an oscillating quartz, but no morphological analysis is shown. A lower value of $1 \mathrm{ML}$ for the critical thickness has also been found by Auger spectroscopy studies for gold evaporated on clean Si (111) at room temperature [16]. Experiments of minute amounts of gold deposited on $\mathrm{NaCl}$ demonstrated that a considerable surface mobility and coalescence is present also at room temperature. Since the diffusion coefficient of gold in silicon cannot explain this rapid interdiffusion, it was concluded that the intermixing of gold into the silicon was a chemically driven process. In the same paper, TEM analysis has been indicated as an improper technique to study the early stages of the deposition of gold on Si because early film growth can be detected only after the adatoms have formed small metal nuclei with sizes exceeding the resolving power of the technique. The top region of the interacted material has been investigated too, and in literature, a controversy is present, some papers referring to an alloy [18], some to a gold-rich material [15], others to a Si rich one [19]. The interaction can be inhibited if the Si surface is covered by $\mathrm{SiO}_{2}$ or impurities such as carbon, water, or oxygen prior to the Au deposition [15]. The effects of the substrate orientation has been explored and the (100) Si surface exhibits higher interactions rates than the (111), due to the larger Si dangling bonds density, available for bonding to $\mathrm{Au}$, present in the first [19]. The role of the deposition technique has been investigated too, and it has been found that in sputtered samples, the interaction is more active than in evaporated films due to the knock off mechanism inferred by the energetic ions during the sputtering on the Si surface, which remove more efficiently oxide residuals and improves the $\mathrm{Au} /$ Si contact [19].

Despite the large amount of works present in literature on the $\mathrm{Au} / \mathrm{Si}$ system and its changes with annealing process, a detailed morphological and structural characterization at the nanometer level of the room temperature evolution of a few ML of gold, i.e., a discontinuous film, deposited on $\mathrm{Si}$ is still missing.

In this paper, a study on gold nanodots deposited by sputtering over (100) Si substrates and after more than $2 \times$ $10^{3} \mathrm{~h}$ of aging at room temperature is presented. The samples are stored in air at temperature between 20 and $23{ }^{\circ} \mathrm{C}$ and humidity of about $40 \%$. The samples have been prepared to avoid any other thermal budget, included that necessary to prepare the specimen for TEM analysis, and the first characterization started just a few hours after the deposition. We used bright field TEM and diffraction analysis to get several snapshots of the changes in the shape, crystallography, and phase of the deposited and evolved nanostructures at different aging times. The kinetics have been quantified by measuring the nanostructures size and density and the Si substrate areas covered by gold. A high statistics has been guaranteed by the usage of digital analysis tools of the TEM micrographs. We demonstrate that the $\mathrm{Au} / \mathrm{Si}$ phase after deposition can be modified by the specimen preparation and by the room temperature exposure in terms of shape, density, composition, thermodynamic phase, and crystallography. We also demonstrate that TEM analysis, if properly prepared, is essential to reveal the morphology of the islands and to distinguish among the different phases to which the deposited material undergoes.

\section{Experimental}

The samples have been prepared by using two different procedures depicted in Fig. 1a, b. In the first case, Fig. 1a, p-type Si substrates were subjected to a chemical etching in HF buffered solution $(7 \%)$ to remove the native oxide. Immediately after cleaning, the samples were introduced into a magnetron sputtering chamber Emitech K550X (Emitech Limited, Kent, UK), with $7 \times 10^{-5}$ mbar of base pressure, $6 \times 10^{-3}$ mbar of deposition pressure in $\mathrm{Ar}$, for the deposition of gold at $15 \mathrm{~mA}$ of gun current and for $30 \mathrm{~s}$. No intentional heating was used during gold deposition. The deposited equivalent gold thickness was measured by an oscillating quartz inside the chamber and resulted equal to $2 \pm 0.1 \mathrm{~nm}$. RBS analysis provided a measurement of the deposited total amount equal to $(1.2 \pm 0.1) \times$ $10^{16} \mathrm{~cm}^{-2}$. This value corresponds to about 7.9 ML of gold. After the gold deposition, the samples have been thinned for the TEM analysis by mechanical grinding from the back and ion milling polishing, which is made from the back of the sample at 
$3 \mathrm{keV}$ with Ar. During the whole thinning procedure, the maximum temperature experienced by the specimen is $150^{\circ} \mathrm{C}$. In the second case, Fig. 1b, the p type Si substrates were first thinned as for the TEM analysis and then were subjected to the HF etch and gold deposition with the same conditions detailed above. Morphological and structural characterization were performed in both cases by TEM in planar view, by using a JEOL JEM 2010F (JEOL Ltd., Tokyo, Japan) microscope with a field emission gun operating at $200 \mathrm{kV}$ accelerating voltage. The samples realized with the procedure in Fig. 1a were analyzed after the TEM preparation, while the samples prepared like in Fig. $1 \mathrm{~b}$ were analyzed right after the gold deposition and after $330 \mathrm{~h}, 1 \times 10^{3} \mathrm{~h}$, and $2.4 \times 10^{3} \mathrm{~h}$. All this time, these samples have been stored in air at temperature ranging between 20 and $23{ }^{\circ} \mathrm{C}$ and humidity of about $40 \%$. The TEM images were digitally analyzed by a Digital Micrograph tool and were transformed in a black and white image with a procedure reported in ref. [20]. The transformed images have then been analyzed, and the corresponding nanostructure areas have been measured. In case of Au nanodots, their radius has been taken as $r_{\mathrm{d}}=\sqrt{\frac{A_{\mathrm{d}}}{\pi}}$, where $A_{\mathrm{d}}$ is the area of the dot. For every type of sample, a statistics of at least 100 dots has been collected.

\section{Results and discussion}

Figure $2 \mathrm{a}$ presents the TEM analysis in planar view of a sample sputtered with the procedure indicated in Fig. 1a, i.e., first, the HF etch plus gold deposition and then the TEM specimen preparation. Two zones can be identified: white regions representing the Silicon substrate and black regions that represent the gold nanodots. Figure $2 b$, c presents TEM in planar view of the as deposited sample obtained as in Fig. 1b, i.e., the HF etch plus gold deposition performed after the TEM thinning. The analysis was performed on the sample a few hours after the deposition. Three zones can be identified: white regions representing the silicon substrate, black regions that represent gold nanodots and an intermediate zone of gray color. In Fig. 2c, d the corresponding electron diffraction patterns of the samples imaged in Fig. 2a, b, respectively, are shown. In Fig. 2c, the blue square is superimposed to the pattern of the (100) Si substrate zone axis. The indexes refer to the Si spots 004 and 022 . The Au spots, indicated by the red indexes, correspond to two Au patterns rotated by $90^{\circ}$ relative to each other with the same zone axis [110] on Si [100]. As observed, the diffraction analysis shows an in-plane alignment of the $\mathrm{Au}$ nanostructures. The orientation relationship is $\mathrm{Au}(110)|| \mathrm{Si}(100)$ with $\mathrm{Au}[002] \mid \mathrm{Si}[022]$ and $\mathrm{Au}[002]|| \mathrm{Si}[02$ $2]$. Figure $2 d$ shows the diffraction pattern relative to the sample prepared with the innovative procedure, i.e., by thinning the specimen before the Au sputtering. Also, in this case, the blue square indicates the $\mathrm{Si}(100)$ substrate zone axis. The arrows indicate the presence of rings typical of Au nanodots randomly oriented between each other and also with respect to the Si substrate. All the diffraction patterns have been taken from an area of $500 \times 500 \mathrm{~nm}$. The diffraction signal corresponding to the amorphous regions is not visible because it is covered by the ones of the randomly oriented dots.

High resolution TEM analysis (not shown here) coupled to diffraction analysis indicates that the cloudy gray region corresponds to an amorphous mixture of gold and silicon, thus
Fig. 1 Schematics depicting the two procedures followed for the synthesis of the samples. a $\mathrm{Si}$ substrates have been HF etched and subjected to the gold sputtering deposition, and then to the TEM thinning procedure. $\mathbf{b} \mathrm{Si}$ substrates have been thinned for the TEM analysis before the HF and gold sputtering deposition

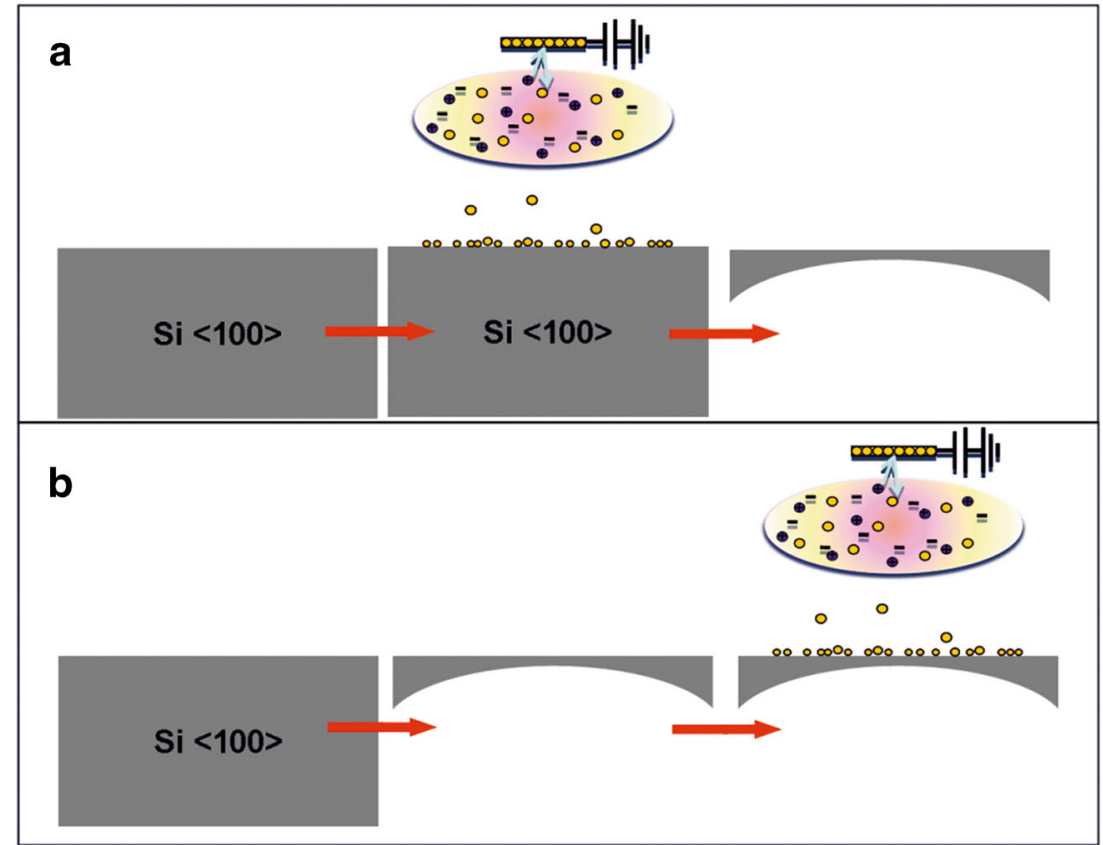


Fig 2 a Bright field TEM in planar view of the sample obtained by using the synthesis procedure indicated in Fig. 1a, i.e., first, the gold sputtering on $\mathrm{Si}$ (100) and then specimen thinning. b Bright field TEM micrograph in planar view of the sample prepared by using the synthesis procedure indicated in Fig. 1b, i.e., first TEM specimen preparation and then HF etch plus gold deposition. c Diffraction pattern of the sample imaged in a, c diffraction pattern of the sample imaged in $\mathbf{b}$
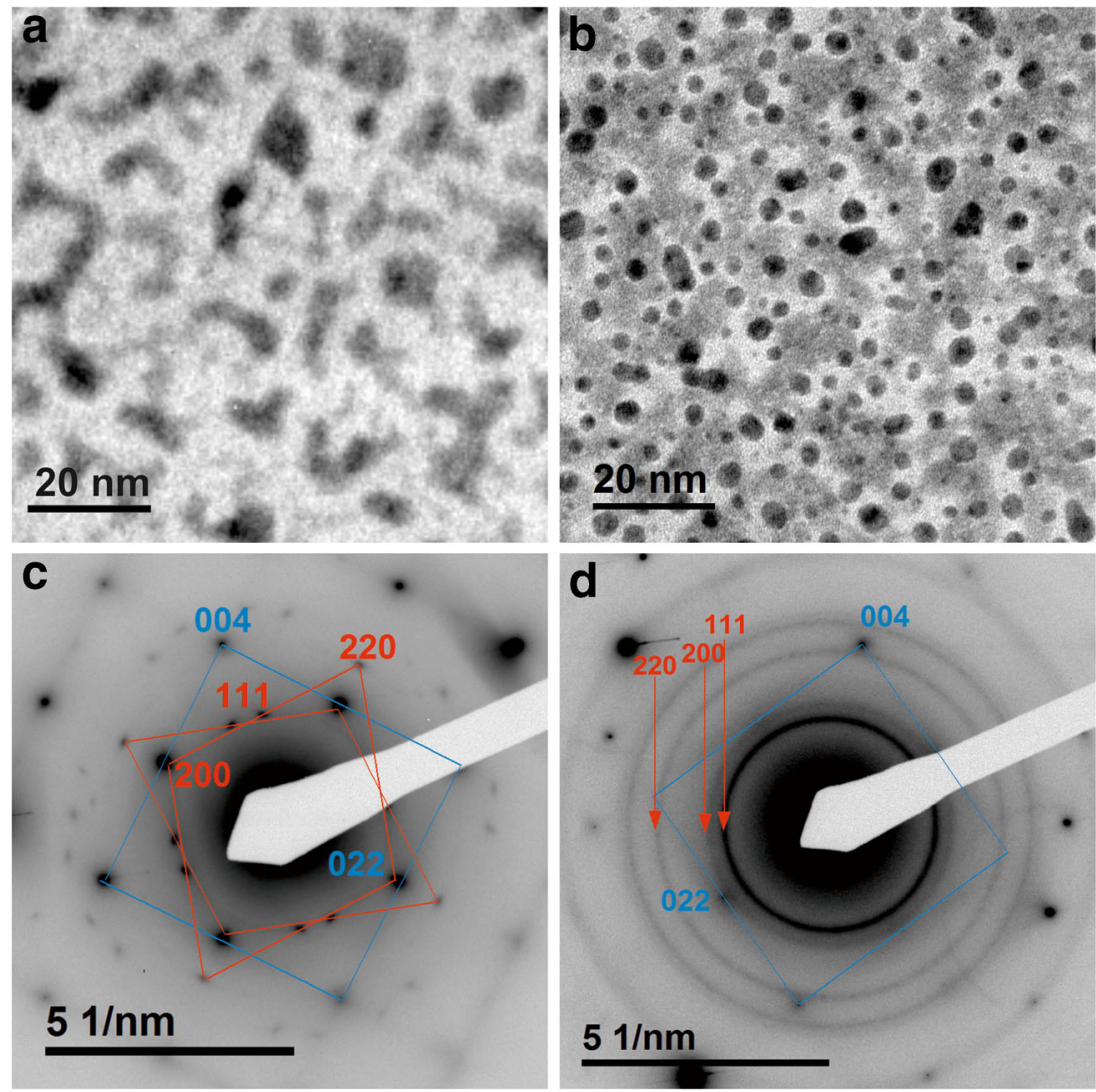

indicating that here gold has intermixed with silicon. Since the characterization is ex situ, it is not possible to establish whether the interaction has proceeded during the deposition because of the energetic plasma, or right after the deposition because of the few hours of room temperature exposure. However, it is reasonable to think that the plasma has contributed to it, as in case of sputtered continuous films [15], although with differences due to the discontinuity of the Au layer which will be discussed in the following. We can conclude that the process performed during the TEM preparation in the case of Fig. 1a modified the original Au morphological and structural characteristics. For this reason, all the results reported in the following have been obtained only by using the procedure in Fig. 1b, i.e., TEM specimen preparation before than the HF plus gold deposition.

Figure 3 shows the TEM micrographs in bright field conditions taken during the time evolution of the sample prepared as in Fig. $1 \mathrm{~b}$ and analyzed a few hours after the deposition (Fig. 3a), after 14 days (Fig. 3b), 43 days (Fig. 3c), and 99 days (Fig. 3d). The first micrograph shows as in Fig. 2c two phases of gold: Au nanodots, the dark gray circular areas indicated by the red arrows, and amorphous regions of intermixed $\mathrm{Au} / \mathrm{Si}$, the large cloudy gray regions highlighted with the orange arrows. The interaction between $\mathrm{Si}$ and $\mathrm{Au}$ is known in literature for thin continuous films annealed at $T<150{ }^{\circ} \mathrm{C}[14,15]$ and will be discussed in the following by comparing the previous results to the new ones found in this work at room temperature [13, 21], while the agglomeration of gold in nanodots is presumably due to some local residual or regrown $\mathrm{Si}-\mathrm{O}$ bonds or $\mathrm{C}$ atoms that prevent the $\mathrm{Si} / \mathrm{Au}$ intermixing [21]. From a preliminary observation of the four micrographs in Fig. 3, it is possible to understand that the evolution of the gold nanodots does not show drastic changes, while the amorphous $\mathrm{Au} / \mathrm{Si}$ agglomerates, corresponding to the gray areas, evidence a morphological modification because they shrink in density and size leaving room to Si uncovered areas. Furthermore, after 43 days of aging, some of the cloudy areas appear darker than in the as deposited and present a contrast more similar to the one of the Au dots. In some cases, these areas (like the ones indicated by the green arrows) exhibit the fringes typical of a crystalline phase lying over a crystalline substrate, indicating that these nanostructures after aging are crystalline. This result demonstrates that to ensure the accuracy of the results, besides than properly preparing the sample for analysis, it is crucial to analyze the samples immediately after the gold deposition. The TEM micrographs have been analyzed by a digital elaboration to measure the size of the dots and of the interacted regions, by averaging on at least 100 objects for each condition. The next results refer to the characterization performed on the nanodots first, and then to the amorphous agglomerates. Figure 4 shows the size distribution of the Au nanodots as a function of their radius for the several days of observation, and Table 1 reports the measured data 
Fig. 3 TEM micrographs in bright field conditions reporting the evolution of the sample during the aging in air at room temperature after a few hours (a), $330 \mathrm{~h}(\mathbf{b}), 1 \times 10^{3} \mathrm{~h}(\mathbf{c})$, and $2.4 \times$ $10^{3}$ (d) of storage. The colored arrows indicate the different materials: Au dots (red arrows) and $\mathrm{Au} / \mathrm{Si}$ cloudy gray zones (orange arrows). The green arrows indicate the fringes inside some of the aligned $\mathrm{Au}$ nanostructures, formed after segregation
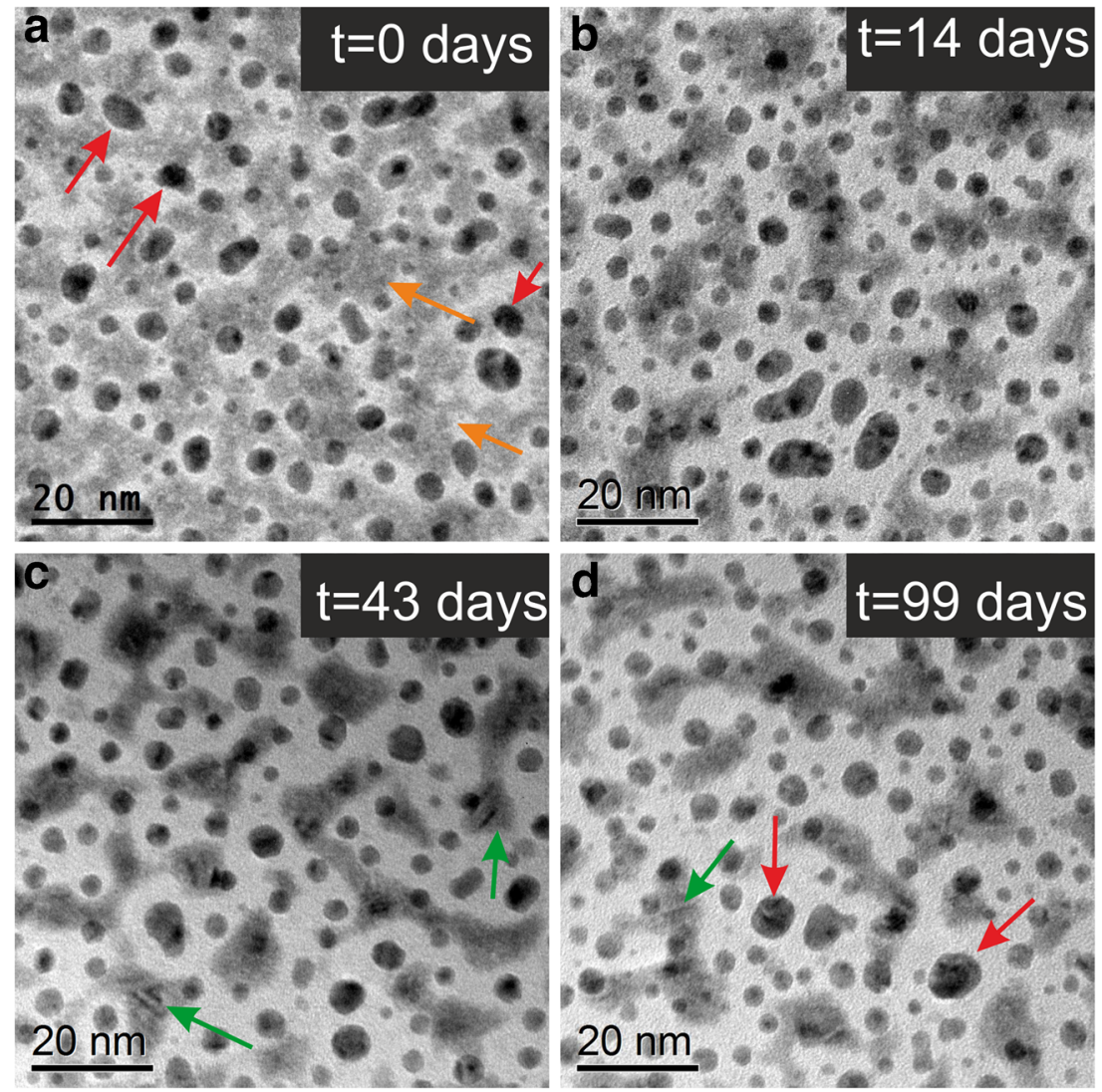

about the average nanodot radius, defined as explained in the "Experimental" section by approximating the shape planar projection of the dots to circles, and their surface density.

From the graph and the data reported in Table 1, we can say that the size distribution of the Au nanodots as a function of the radius presents a shape that is maintained all over the time with small differences in the peak value and in the centroid.

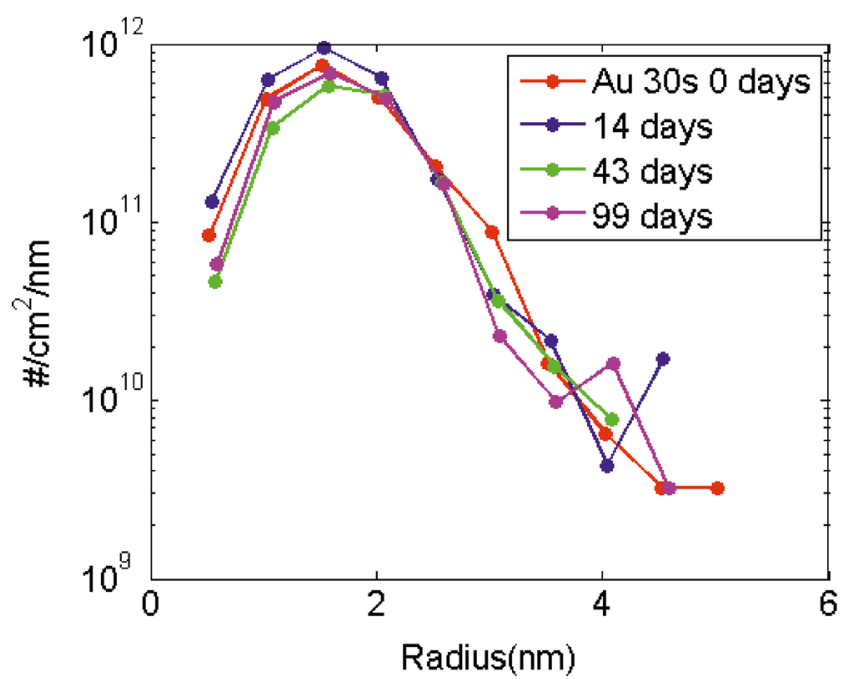

Fig 4 Gold dot radius distribution for the as deposited samples (red) and for the sample stored for $330 \mathrm{~h}$ (blue), $1 \times 10^{3} \mathrm{~h}$ (green), and $2.4 \times 10^{3} \mathrm{~h}$ (magenta) in air
The quantitative analysis of the data then confirms the previous observations, i.e., the nanodots morphological characteristics do not show appreciable evolution over time, supporting the hypothesis that at the interface, the presence of residual or regrown $\mathrm{SiO}_{\mathrm{x}}$, or even carbon atoms, have inhibited the intermixing between $\mathrm{Au}$ and $\mathrm{Si}$.

The previous data refer to the characteristics of the agglomerated Au nanodots. The regions where the gold is mixed with Si have been analyzed too. Since their shape cannot be approximated as circular, the parameter chosen to investigate their properties has been their area. The percentage of area covered with $\mathrm{Au} / \mathrm{Si}$ intermixed nanostructures, $\Theta_{\mathrm{g}}$, has been obtained from the difference of two quantities measured experimentally: the coverage of the substrate with agglomerated gold nanodots, $\Theta_{\mathrm{d}}$, and the percentage of uncovered substrate $\Theta_{\mathrm{s}}$ (the white areas present in the TEM micrographs of Fig. 3).

Table 1 Average radius and dot surface density as a function of the evolution time

\begin{tabular}{lll}
\hline $\begin{array}{l}\text { Evolution time } \\
\text { (days) }\end{array}$ & $\begin{array}{l}\text { Dot radius } r_{\mathrm{d}} \\
(\mathrm{nm})\end{array}$ & $\begin{array}{l}\text { Dot density } \\
\left(\# / \mathrm{cm}^{2}\right)\end{array}$ \\
\hline 0 & $1.7 \pm 0.6$ & $2.2 \times 10^{12}$ \\
14 & $1.6 \pm 0.6$ & $2.6 \times 10^{12}$ \\
43 & $1.8 \pm 0.6$ & $1.7 \times 10^{12}$ \\
99 & $1.7 \pm 0.6$ & $1.9 \times 10^{12}$ \\
\hline
\end{tabular}


We calculated the total Au coverage, $\Theta_{\mathrm{G}}$, as the difference between 1 and the fraction of uncovered Si substrate $\Theta_{\mathrm{s}}$. After this, $\Theta_{\mathrm{g}}$ has been calculated as $\Theta_{\mathrm{G}}-\Theta_{\mathrm{d}}$. Figure 5 reports the results on $\Theta_{\mathrm{d}}$ (light blue square symbols), $\Theta_{\mathrm{s}}$ (diamond red symbols), $\Theta_{\mathrm{G}}$ (green circles), and $\Theta_{\mathrm{g}}$ the region of interacted $\mathrm{Au} / \mathrm{Si}$ (magenta triangles).

The first consideration that can be inferred from Fig. 5 is that $\Theta_{d}$ is approximately constant, as anticipated by the results of Fig. 4. Instead, if we look at the amount of gold that has intermixed with $\mathrm{Si}, \Theta_{\mathrm{g}}$, it decreases rapidly in the first 15 days and then tends to saturate. The fact that the quantity of $\mathrm{Au}$ interacted with $\mathrm{Si}$ decreases after aging will be discussed later in the paper, in the comments to Fig. 7.

The observation of the samples through the weeks after the deposition has also highlighted certain aspects of the crystallographic orientations of the aged material. The Fig. $6 a, b, c$ reports the diffraction analysis for the $330 \mathrm{~h}(\mathrm{a}), 1 \times 10^{3} \mathrm{~h}(\mathrm{~b})$, and $2.4 \times 10^{3} \mathrm{~h}(\mathrm{c})$ aged samples. The diffraction patterns for all the samples have been taken off axis to avoid that the spots relative to the $\mathrm{Si}$ substrate, much more intense than the $\mathrm{Au}$ diffraction rings and spots, would saturate the image intensity and obscure the Au diffraction features. Figure 6a shows a sample after 14 days of aging. The sample area where the diffraction pattern is taken is about $500 \times 500 \mathrm{~nm}$. In this area, the sample resulted to be bent, and this fact has created different diffraction spots with respect the expected ones.

A blue square is superimposed to all the diffraction patterns as a guide to the eyes to identify Si spots. The concentric circles, present in Fig. 6a, are formed by the presence of the randomly oriented Au nanodots that behave as powders. In the diffraction pattern relative to the 43rd (6b) and 99th (6c) days, the weak

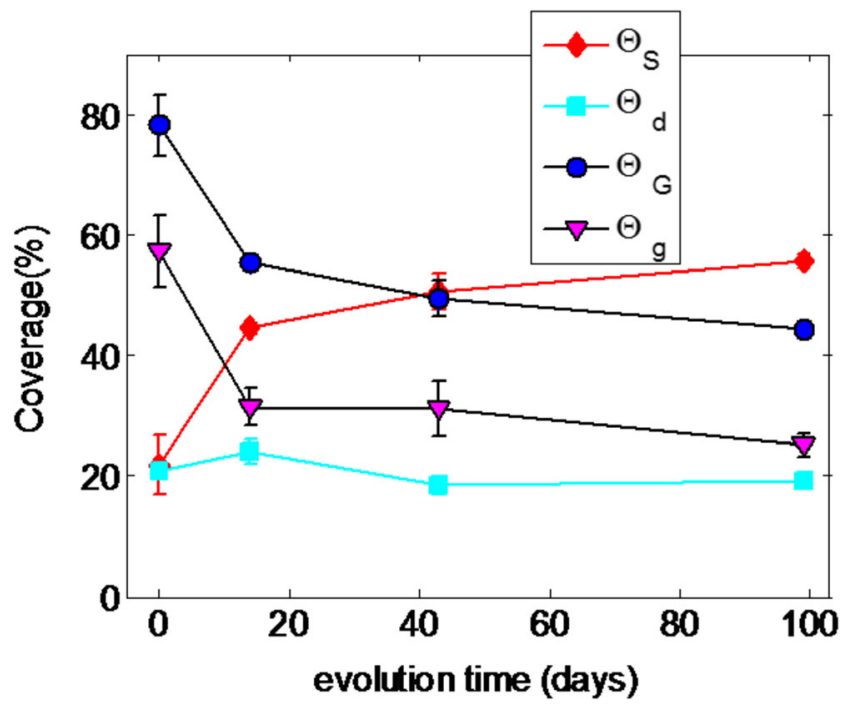

Fig. 5 Measured data on the percentage of uncovered substrate $\Theta_{\mathrm{s}}$ (diamond red symbols) and on the coverage of gold nanodots $\Theta_{\mathrm{d}}$ (cyan square symbols) as a function of evolution time; calculated values of coverage as a function of evolution time for the total $\mathrm{Au}, \Theta_{\mathrm{G}}$ (blue circles), and interacted gold, $\Theta_{\mathrm{g}}$ (magenta triangles)

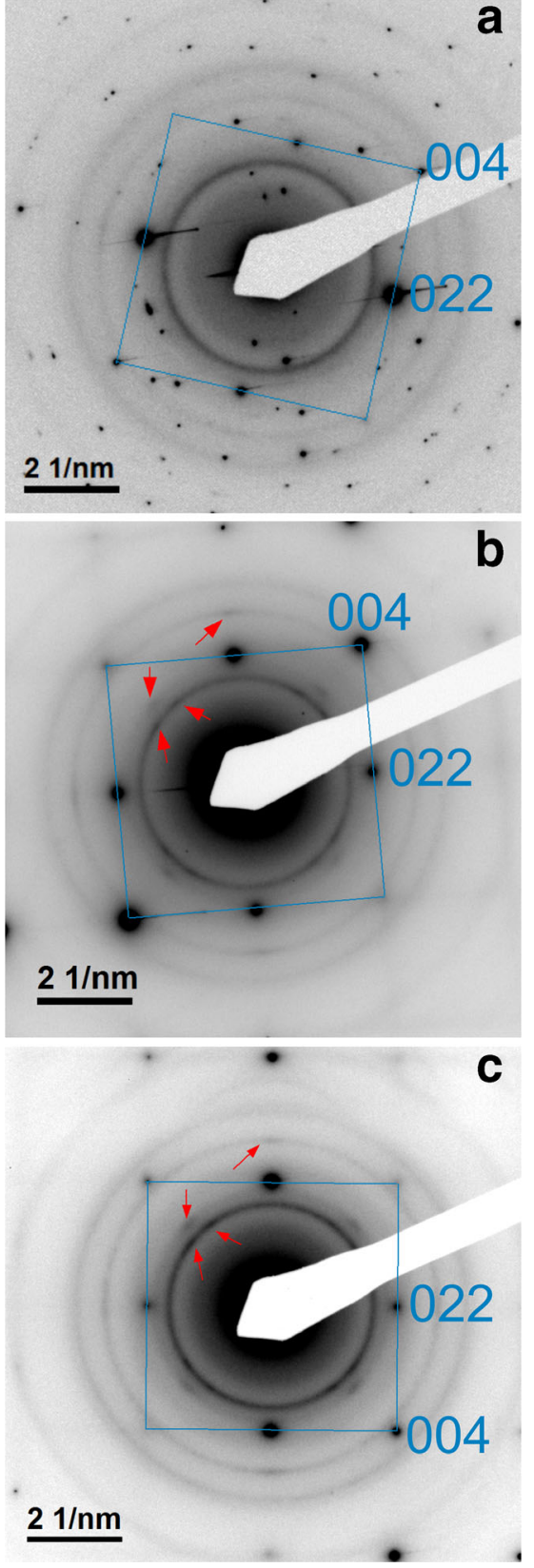

Fig. 6 Diffraction patterns of the samples aged for 14 (a), 43 (b), and 99 days (c). In the diffraction pattern relative to the 43rd and 99th day, the weak spots (identified by the red arrows) correspond to the orientation relationship $\mathrm{Si}(\mathrm{Au}(110) \| \mathrm{Si}(100)$ with $\mathrm{Au}[002]|| \mathrm{Si}[022]$ and $\mathrm{Au}[002]|| \operatorname{Si}[02-2])$

spots (identified by the red arrows) correspond to two $\mathrm{Au}$ patterns rotated by $90^{\circ}$ relative to each other with the same zone axis [110] on $\mathrm{Si}$ [100]. This is a similar result to the alignment previously observed in the case of the sample prepared by sputtering $\mathrm{Au}$ before the TEM thinning procedure, presented in Fig. 2c. The orientation relationship is $\mathrm{Au}(110)|| \mathrm{Si}(100)$ with $\mathrm{Au}[002] \mid \mathrm{Si}[022]$ and $\mathrm{Au}[002] \mid \mathrm{Si}[02-2]$.

The experimental results coming from the diffraction patterns coupled to those from the bright field analysis indicate an 
evolution of the sample toward the crystalline phase, the presence of the ring in every diffraction figure is due to the nanodots that are randomly oriented, and the interacted alloyed phase that is amorphous in the as deposited sample presents a phase transition demonstrated by the presence of weak spots that indicated the alignment. After 99 days of aging, experimental data shows the evidence of a transition of the material. The gold atoms from the alloy, tend to segregate in subsurface $\mathrm{Au}$ clusters similar to the "inert" ones, but are preferentially oriented with silicon. Regions of intermixed alloys are however still present in the sample.

In summary, the morphological and structural data have thus shown that gold deposited by sputtering on clean $\mathrm{Si}$ shows two phases: gold agglomerated as nanodots of a few nanometers in size and amorphous $\mathrm{Au} / \mathrm{Si}$ agglomerates. The latter evolves at room temperature and tends to form smaller and fewer crystalline regions of pure gold aligned with some crystallographic orientations of the silicon substrate.

An explanation of the results obtained in this study relatively to the $\mathrm{Si} / \mathrm{Au}$ intermixing is provided by a work of J. K. $\mathrm{Bal}$ and S. Hazra [21]. They studied the room temperature diffusion of continuous thin layers of gold on Si (001) substrates and have observed that in samples whose surface was initially treated with HF to remove the native oxide, as in our case, gold interacts with silicon diffusing through the surface. The amount of diffusing gold saturated after about 2 weeks from the date of deposition due to the regrowth of an oxide layer, which slows or prevents the intermixing Au-Si. The saturation time and the decrease in the interaction kinetics are very similar to our case. The literature data refer however to the characterization of continuous gold layers diffused in $\mathrm{Si}$ and does not present data on the morphological and structural characteristics of the nanostructures.

Relatively to the Au-Si interaction, it is known in literature that the intermixing between $\mathrm{Au}$ and $\mathrm{Si}$ even at low temperatures, produces a gold-rich (70\%) amorphous surface alloy [22, 23]. Moreover, Kim al. [24] proposed a structural model, indicating the arrangement of atoms in the layers as they are deposited. The proposed model shows that at the interface between the deposited material and the substrate, the system $\mathrm{Au} / \mathrm{Si}$ tends to reach the concentration of a $50-50$ of compound Au-Si. Once reached this level, the system tends to preserve this condition, so it behaves as a "stable" condition.

In the as deposited sample, we expect to find $1 \mathrm{ML}$ at the interface with $\mathrm{Si}$ with a concentration of $50 \%$; in the following discussion, we neglect this contribution. We start from the total amount of deposited gold and separate the contribution of the crystalline surface dots and the amorphous subsurface intermixed alloys. The amount of gold in the clusters is directly calculated from the TEM data, from the measured radius of each dot, by considering that gold dots have a circular shape, and from the equivalent gold thickness, by using the standard gold bulk density (because the dots present random crystal orientations). The amount of gold which is contained in the amorphous $\mathrm{Si}-\mathrm{Au}$ alloy is calculated by subtracting the quantity of gold in the crystalline dots from the total amount of gold. By taking into account that the amorphous zones, in the as deposited case, present a concentration of $70 \%$ Au rich (by considering the literature results from AES analysis [22]), we calculate the equivalent thickness of the amorphous $\mathrm{Au}-\mathrm{Si}$ alloy and report the results in Fig. 7. An analogous procedure has been carried for the 99th day of aged sample, but in this case, considering that the sample is made of crystalline pure gold, we considered a $100 \% \mathrm{Au}$ concentration. For the intermediate samples, the 14th and 43rd days of aging, a concentration from 70 to $100 \%$ has been taken into account. In Fig. 8, we present the data relative to the calculated thickness for each case. The green square represent the thickness calculated by using $70 \%$ for gold concentration, and the blue square represent the data calculated by using the $100 \%$ concentration for the gold dots. The rectangles for the 15 th and 43rd days indicate the possible thickness of the nanophases if the concentration of gold in the alloy goes from $70 \%$ (green shade) to $100 \%$ (blue shade). In the graph, the amount of gold in form of nanodots is not plotted because it does not change over time.

The peculiarity of the analysis performed in the present work relies on the capability to quantify only the data relative to the Au-Si intermixed compound, distinguishing from the inert surfacial nanodots by means of the TEM analysis in planar view which allows to quantify the effective area covered by the gold and alloyed islands. Moreover, thanks to the

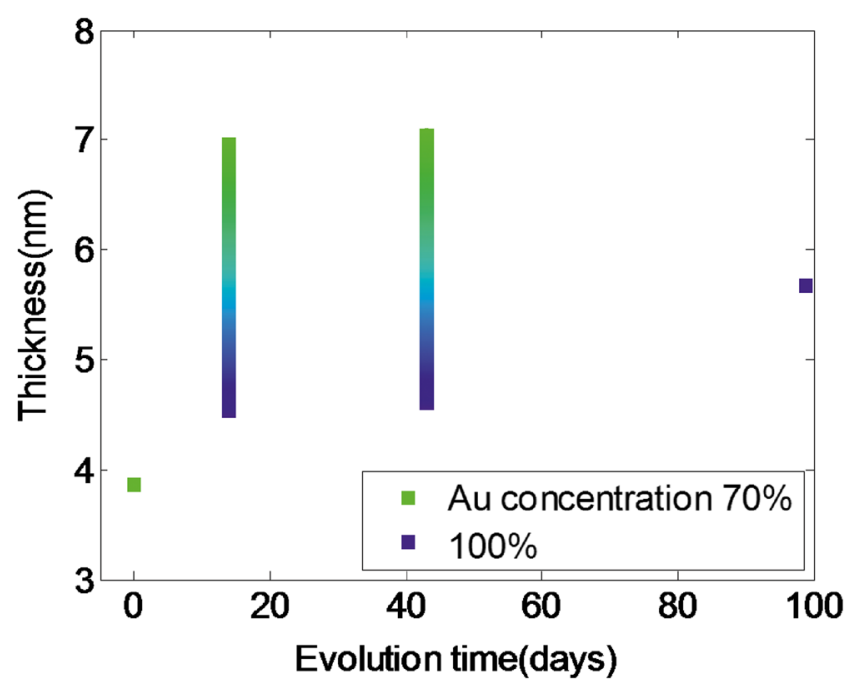

Fig. 7 Calculated equivalent thickness of the amorphous $\mathrm{Au} / \mathrm{Si}$ alloys, during the aging (from the as deposited case to the aged samples) and the transition to the crystalline $\mathrm{Au}$ dots. The green square represent the thickness calculated by using $70 \%$ for gold concentration and the blue square represent the data calculated by using the $100 \%$ concentration for the gold dots. The rectangles for the 15th and 43rd days indicate the possible thickness of the nanophases if the concentration of gold in the alloy goes from $70 \%$ (green shade) to $100 \%$ (blue shade). In the graph, the amount of gold in form of nanodots is not reported because it does not change over time 
a

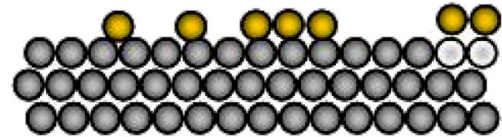

b

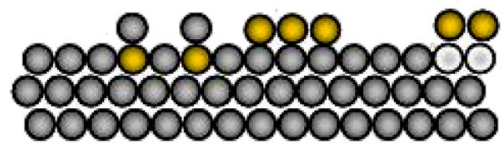

C
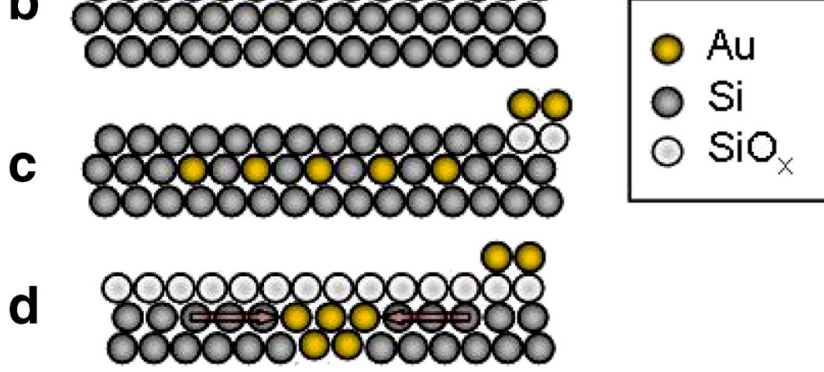

Fig. 8 Schematic summarizing the gold nanocluster characteristics during deposition (a to c) and aging (d). a Gold deposited on clean Si. b Au$\mathrm{Si}$ interdiffusion all over the surface except where there is residual or regrown $\mathrm{SiO}_{\mathrm{x}}$. $\mathrm{c}$ The intermixing creates a surface Si layer and subsurface amorphous agglomerates of $\mathrm{Au}-\mathrm{Si}$, forming a stable stoichiometry of $70 \%$. d After the deposition and during the weeks later, Au starts to diffuse segregating in crystalline dots aligned with some crystallographic orientations of Si and occupying smaller and less dense areas

special specimen preparation, Au does not undergo to thermal treatment, except the $20-23{ }^{\circ} \mathrm{C}$ temperature exposure, and does not modify its original morphological characteristics. It should be noted that the data relative to the aged samples in Fig. 7, takes only into account the areal projection of the gold alloys and does not take into account their thickness, so they have to be understood as an estimation. From our data, we can then conclude that the $\mathrm{Au} / \mathrm{Si}$ interdiffusion in the as deposited samples takes place also in case of nanoislands, when in contact with Si producing amorphous alloys of about 70:30 $\mathrm{Au}: \mathrm{Si}$ composition and that after aging, it tends to the $100 \%$ $\mathrm{Au}$ composition realigned in both $<100>$ and $<200>$ orientations. From Figs. 5 and 7, it can also be concluded that the coverage of Au interacted with Si decreases after aging, and this is correlated to the relatively slow spinodal decomposition kinetics occurring at RT (which is well below the eutectic temperature $T_{\mathrm{e}}=363{ }^{\circ} \mathrm{C}$ ). Considering the equilibrium phases diagram and the $\mathrm{Au}-\mathrm{Si}$ system, this process should cause after a certain time the complete separation of the mixed $\mathrm{AuSi}$ region in the two elemental phases which nucleate (in our case only pure $\mathrm{Au}$ nucleates) and grow at expenses of the intermixed region. However, the shrinking of the amorphous $\mathrm{Au}-\mathrm{Si}$ nanostructures in size and density can be followed in our aging experiment since the mobility of the phase boundary is low due to the small temperature and the surface proximity.

Figure 8 is a schematic summarizing the results found in this work, supported by literature findings. First, gold is deposited on clean $\mathrm{Si}$, and the gold atoms are randomly placed over the surface (a). Gold starts mixing with Si by interdiffusion. Due to some residual or regrown local $\mathrm{SiO}_{\mathrm{x}}$ the gold deposited on the oxide does not interact with $\mathrm{Si}$ and form nuclei for stable crystalline dots (b). The intermixing creates a surface Si layer and subsurface amorphous islands of mixed $\mathrm{Au}-\mathrm{Si}$, which tend to form a stable stoichiometry of $70 \%$ [22] (c). Figure $8 \mathrm{a}, \mathrm{c}$ refer to processes happening during the growth. After the sample is taken out of the chamber, gold starts to diffuse segregating in crystalline islands aligned with some crystallographic orientations of $\mathrm{Si}$ and occupying smaller and less dense areas through a process similar to ripening. The exchange of gold atoms between the crystalline dots and the subsurface agglomerates is inhibited by the presence of contaminants (d).

If we compare the sample characteristics after 99 days of aging, Fig. 3d, and the sample prepared by using the standard TEM preparation, Fig. 2a, we see that they are similar, i.e., the areas covered by the interacted $\mathrm{Au} / \mathrm{Si}$ phase are similar in size and shape and both present crystalline Au segregated regions. The only difference is that the gold clusters in the second case present a smaller density and are placed over the interacted regions. This suggests that the $150{ }^{\circ} \mathrm{C}$ annealing or the ion milling, can have promoted the "interaction" between the agglomerated clusters and the subsurface mixed phases, meaning that the barrier in this case has been removed or it was absent. However, the result let to understand that TEM, with the proper specimen preparation, is essential to characterize small amounts of alloyed $\mathrm{Au} / \mathrm{Si}$ islands, in the early film growth even if they are still amorphous and before the elementary surface processes, such as surface diffusion, chemical reaction, or phase transition occur. The presence of islands growth of gold and gold alloys, and their evolution helps to explain also the controversy present in literature about the composition of the top part of the intermixed phase. The data indeed depend (1) on the initial gold coverage: for low coverage the Si signal coming from the matrix prevails, while for high coverage the signal of the pure and alloyed $\mathrm{Au}$ is present; (2) on the aging time: in the "fresh" case, both amorphous alloyed and crystalline pure Au phases (with random orientations) can be found; after aging, predominantly crystalline phases (either random or aligned) of pure gold immersed in a silicon matrix are contributing, together with some residual amorphous phases.

\section{Conclusions}

In this manuscript, a study on the morphological and structural characteristics of gold nanodots deposited by sputtering on $\mathrm{Si}$ $<100>$ substrates and their time evolution during storage at room temperature is reported. This material is interesting for a number of applications such as light harvesting through plasmonic effects, metallic contacts in standard miniaturized devices, and catalysis of Si-NWs. As it is known in literature, the performance of all these materials in the final devices depend on the $\mathrm{Au} / \mathrm{Si}$ system morphology and characteristics. The time 
evolution at room temperature of gold sputtered samples is monitored in this paper by TEM analysis, from a few hours up to $2.4 \times 10^{3} \mathrm{~h}$ after deposition. The variation of density and size of gold nanodots are obtained averaging on a large statistics.

The characterization shows the presence of two phases for $\mathrm{Au}$ nanostructures after deposition, i.e., (1) gold nanodots with diameter of few nanometers that do not have interaction with substrate and (2) amorphous nanophases of intermixed $\mathrm{Au} / \mathrm{Si}$. The structural characteristics of gold nanodots do not show a sensitive variation with time, attributed to the fact that locally, the presence contaminants slows down or inhibits the interdiffusion with $\mathrm{Si}$. Au/Si amorphous agglomerates evolve, and their coverage rapidly decreases in the first 15 days after the deposition. Diffraction patterns of the interacted regions show that during the evolution, these areas tend to segregate forming pure gold crystalline regions aligned with some crystallographic orientations of $\mathrm{Si}(\mathrm{Au}(110)|| \mathrm{Si}(100)$ with $\mathrm{Au}[002] \| \mathrm{Si}[022]$ and $\mathrm{Au}[002]|| \mathrm{Si}[02-2])$.

We can conclude that the nanostructured Au-Si system evolves rapidly with time and this demonstrates the importance of analyzing the samples immediately after their deposition and without any modification induced by low thermal budget processes.

Acknowledgments The authors wish to thank Markus Italia, Salvatore Di Franco, and Rosa Ruggeri (CNR-IMM) for their valuable work and help during the samples preparation and analysis, and F. Ruffino (University of Catania) for RBS analysis.

Open Access This article is distributed under the terms of the Creative Commons Attribution License which permits any use, distribution, and reproduction in any medium, provided the original author(s) and the source are credited.

\section{References}

1. Atwater HA, Polman A (2010) Plasmonics for improved photovoltaic devices. Nat Mater 9:205-213. doi:10.1038/nmat2629

2. Catchpole KR, Polman A (2008) Plasmonic solar cells. Opt Express 6(26):21793

3. Catchpole KR, Polman (2008) A design principles for particle plasmon enhanced solar cells. Appl Phys Lett 93:191113. doi:10.1063/1. 3021072

4. Sivakov V, Andrä G, Himcinschi C, Gösele U, Zahn DRT, Christiansen S (2006) Growth peculiarities during vapor-liquidsolid growth of silicon nanowhiskers by electron-beam evaporation. Appl Phys A 85:311-315. doi:10.1007/s00339-006-3675-0

5. Irrera A, Pecora EF, Priolo F (2009) Control of growth mechanisms and orientation in epitaxial $\mathrm{Si}$ nanowires grown by electron beam evaporation. Nanotechnology 20:135601. doi:10.1088/0957-4484/ 20/13/135601

6. Garozzo C, Puglisi RA, Bongiorno C, Scalese S, Rimini E, Lombardo S (2011) Selective diffusion of gold nanodots on nanopatterned substrates realized by self-assembly of diblock copolymers. J Mater Res 26:240-246. doi:10.1557/jmr.2010.19
7. Garozzo C, La Magna A, Mannino G, Privitera V, Scalese S, Sberna PM, Simone F, Puglisi RA (2013) Competition between uncatalyzed and catalyzed growth during the plasma synthesis of Si nanowires and its role on their optical properties. J Appl Phys 113:214313. doi: $10.1063 / 1.4809557$

8. Sivakov VA, Brönstrup G, Pecz B, Berger A, Radnoczi GZ, Krause M, Christiansen SH (2010) Realization of vertical and zigzag single crystalline silicon nanowire architectures. J Phys Chem C 114:37983803. doi:10.1021/jp909946x

9. Schmidt V, Senz S, Gösele U (2005) Diameter-dependent growth direction of epitaxial silicon nanowires. Nano Lett 5(5):931-935. doi: $10.1021 / \mathrm{nl} 050462 \mathrm{~g}$

10. Huang Z, Shimizu T, Senz S, Zhang ZX, Lee W, Geyer N, Gösele U (2009) Ordered arrays of vertically aligned [110] silicon nanowires by suppressing the crystallographically preferred $<100>$ etching directions. Nano Lett 9:2519. doi:10. $1021 / \mathrm{n} 1803558 \mathrm{n}$

11. Chang S-W, Chuang VP, Boles ST, Thompson CV (2010) Metalcatalyzed etching of vertically aligned polysilicon and amorphous silicon nanowire arrays by etching direction confinement. Adv Funct Mater 20(24):4364 4370. doi:10.1002/adfm.201000437

12. Milazzo RG, D'Arrigo G, Spinella C, Grimaldi MG, Rimini E (2012) Ag-assisted chemical etching of (100) and (111) n-type silicon substrates by varying the amount of deposited metal. J Electrochem Soc 159(9):D521-D525. doi:10.1149/2.008209jes

13. Akhtari-Zavareh A, Li W, Maroun F, Allongue P, Kavanagh KL (2013) Improved chemical and electrical stability of gold silicon contacts via epitaxial electrodeposition. J Appl Phys 113:063708. doi:10.1063/1.4792000

14. Hiraki A, Lugujjo E, Mayer JW (1972) Formation of silicon oxide over gold layers on silicon substrates. J Appl Phys 43:3643. doi:10. 1063/1.1661782

15. Okuno K, Ito T, Iwami M, Hiraki A (1980) Presence of critical Aufilm thickness for room temperature interfacial reaction between $\mathrm{Au}($ film) and Si(crystal substrate). Solid State Commun 34:493497. doi:10.1016/0038-1098(80)90659-6

16. Andersson TG (1982) The initial growth of vapour deposited gold films. Gold Bull 15(1):7-18. doi:10.1007/BF03216565

17. Hiraki A, Kim SC, Imura T, Iwami M (1979) Si(LMM) Auger

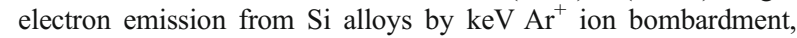
new effect and application. Jpn J Appl Phys 18:1767-1772. doi:10. 1143/JJAP.18.1767

18. Braicovich L, Garner CM, Skeath PR, Su CY, Chye PW, Lindau I, Spicer WE (1979) Photoemission studies of the silicon-gold interface. Phys Rev B 20:5131-5141. doi:10. 1103/PhysRevB.20.5131

19. Chang CA, Ottaviani G (1984) Outdiffusion of Si through gold films: the effects of $\mathrm{Si}$ orientation, gold deposition techniques and rates, and annealing ambients. Appl Phys Lett 44: 901. doi:10.1063/1.94928

20. Puglisi RA, Nicotra G, Lombardo S, Spinella C, Ammendola G, Gerardi C (2005) Partial self-ordering observed in silicon nanoclusters deposited on silicon oxide substrates by chemical vapor deposition. Phys. Rev. B 71 (12) doi:10.1103/PhysRevB.71.125322

21. Bal JK, Hazra S (2007) Interfacial role in room-temperature diffusion of $\mathrm{Au}$ into Si substrates. Phys Rev B 75:20541. doi:10.1103/ PhysRevB.75.205411

22. Hiraki A, Iwami M (1974) Electronic structure of thin gold film deposited on silicon substrate studied by Auger electron and X-ray photoelectron spectroscopies. Jpn J Appl Phys 2(Supplement 2-2):749-752

23. Hiraki A, Shimizu A, Iwami M, Narusawa T, Komiya S (1975) Metallic state of $\mathrm{Si}$ in $\mathrm{Si}$ noble metal vapor quenched alloys studied by Auger electron spectroscopy. Appl Phys Lett 26:57. doi:10.1063/1.88054

24. Kim JH, Yang G, Yang S, Weiss AH (2001) Study of the growth and stability of ultra-thin films of Au deposited on $\operatorname{Si}\left(\begin{array}{lll}1 & 0 & 0\end{array}\right)$ and $\operatorname{Si}\left(\begin{array}{lll}1 & 1 & 1\end{array}\right)$. Surf Sci 475:37-46. doi:10.1016/S0039-6028(00)01059-1 